\title{
Sequential epiretinal membrane removal with internal limiting membrane peeling in brilliant blue G-assisted macular surgery
}

\author{
Ricarda G Schumann, ${ }^{1}$ Arnd Gandorfer, ${ }^{1}$ Kirsten H Eibl, ${ }^{1}$ Paul B Henrich, ${ }^{2}$ \\ Anselm Kampik, ${ }^{1}$ Christos Haritoglou ${ }^{1}$
}

${ }^{1}$ Department of Ophthalmology, Vitreoretinal and Pathology Unit, Ludwig-Maximilians-University, Munich, Germany

${ }^{2}$ Department of Ophthalmology, University Hospital Basel, Basel, Switzerland

\section{Correspondence to}

Dr Ricarda G Schumann, Department of Ophthalmology, Vitreoretinal and Pathology Unit, Ludwig-Maximilians-University, Mathildenstr. 8, Munich 80336, Germany; ricarda.schumann@ med.uni-muenchen.de

Presented in part at the Annual Meeting of the Deutsche Ophthalmologische Gesellschaft, Berlin, Germany, September 2009.

Accepted 30 April 2010 Published Online First 31 July 2010

\section{ABSTRACT}

Purpose To assess the selectivity of brilliant blue G (BBG) staining by analysing the morphological components of unstained and stained tissue obtained during epiretinal membrane (ERM) removal with internal limiting membrane (ILM) peeling in BBG-assisted macular surgery.

Methods Twenty-six surgical specimens were removed from 13 eyes with epiretinal gliosis during vitrectomy using BBG for ERM and ILM peeling. We included eyes with idiopathic macular pucker, idiopathic macular hole and vitreomacular traction syndrome. The dye was injected into the fluid-filled globe. Unstained and stained epiretinal tissue was harvested consecutively and placed into separate containers. All specimens were processed for conventional transmission electron microscopy.

Results The first surgical specimen of all eyes showed no intraoperative staining with BBG and corresponded to masses of cells and collagen. The second surgical specimen demonstrated good staining characteristics and corresponded to the ILM in all patients included. In seven eyes, the ILM specimens were seen with minor cell proliferations such as single cells or a monolayer of cells. Myofibroblasts, fibroblasts and astrocytes were present. In five cases, native vitreous collagen fibrils were found at the ILM. In six of the eyes, ILM specimens were blank.

Conclusion Our clinicopathological correlation underlines the selective staining properties of BBG. The residual ILM is selectively stained by BBG even when a small amount of cells and collagen adheres to its vitreal side. To reduce the retinal exposure to the dye, the surgeon might choose to remove the ERM without using the dye, followed by a BBG injection to identify residual ILM.

\section{INTRODUCTION}

In macular surgery, the intravitreal administration of brilliant blue $\mathrm{G}$ (BBG) became a favourable tool to visualise the otherwise translucent internal limiting membrane (ILM). In contrast to other vital dyes such as indocyanine green (ICG), BBG was shown to have a good safety profile providing satisfactory anatomical and functional postoperative results. ${ }^{1-8}$ Brilliant blue $G$ is now considered the dye of choice to stain the ILM by many vitreoretinal surgeons with respect to its selective staining properties.

In order to perform macular surgery safely and obtain the best possible functional results for the patient, it is of great importance that the surgeon can rely on the staining pattern of BBG. However, in the presence of epiretinal tissue, the dye has no access to the ILM. Therefore, ERM formation may impair sufficient staining of the ILM. Poor staining may result in an incomplete removal of the ILM associated with residual ILM fragments with an undefinite extent of cells and collagen remnants at the vitreal side of the ILM. ${ }^{9}$ ILM remnants may contribute to ERM recurrences or reopening of a macular hole by serving as a scaffold for cellular proliferation. Although vital dyes such as BBG facilitate macular surgery, we need to consider tissue-dye interactions and potential adverse effects associated with these adjuncts.

The differentiation of ultrastructural components of unstained and stained tissue removed by BBG-assisted peeling might have an impact on the intraoperative procedure in macular surgery. Knowing about the staining pattern and the reliability of staining characteristics will help the vitreoretinal surgeon to adjust the use of BBG to situations, in which the dye provides relevant information. We conducted this laboratory investigation to elucidate the morphological correlate of sequentially peeled ERM and ILM specimens after $\mathrm{BBG}$ staining.

\section{PATIENTS AND METHODS}

Thirteen eyes from 13 patients underwent vitrectomy with removal of both the ERM and the ILM as a sequential peeling procedure with double BBG staining during January 2008 and April 2009 at the University Eye Hospital Munich. We included nine patients with idiopathic macular pucker (IMP), two patients with idiopathic macular holes (IMH) and two patients with vitreomacular traction syndrome (VMTS). The study was approved by the local institutional review board and ethics committee.

The surgical technique consisted of a standard three-port pars plana vitrectomy with induction of a posterior vitreous detachment by suction with the vitrectomy probe around the optic nerve head. A sterile $0.2 \mathrm{mg} / \mathrm{ml}$ Brilliant blue $G$ solution $(0.5 \mathrm{ml}, 0.25 \%$, Brilliant Peel; Fluoron $\mathrm{GmbH}$, Neu-Ulm, Germany) was injected into the fluidfilled vitreous cavity over the macular area and washed out immediately. The staining characteristics were assessed by the surgeon. Then, unstained tissue was removed in a first step. This was followed by reinjection of a few drops of BBG. In a second step, tissue stained with BBG was removed and harvested respectively. ERM and ILM 
Downloaded from bjo.bmj.com on January 21, 2014 - Published by group.bmj.com

Table 1 Ultrastructural features of both the first and the second surgical specimens removed consecutively in macular surgery with brilliant blue $\mathrm{G}$ staining

\begin{tabular}{|c|c|c|c|c|c|c|c|c|c|c|c|}
\hline \multirow[b]{3}{*}{ No. of patients } & \multirow[b]{3}{*}{ Diagnosis } & \multicolumn{5}{|c|}{ First surgical specimen, no staining } & \multicolumn{5}{|c|}{ Second surgical specimen, good staining } \\
\hline & & \multirow[b]{2}{*}{ ILM } & \multirow[b]{2}{*}{ Cell proliferation* } & \multicolumn{3}{|c|}{ Collagen } & \multirow[b]{2}{*}{ ILM } & \multirow[b]{2}{*}{ Cell proliferation* } & \multicolumn{3}{|c|}{ Collagen } \\
\hline & & & & NVC & NFC & $\overline{F L S C}$ & & & NVC & NFC & FLSC \\
\hline 1 & $\mathrm{IMH}$ & - & Multilayer & ++ & + & + & Present & Single cells & - & - & - \\
\hline 2 & $\mathrm{IMH}$ & - & Multilayer & + & + & - & Present & None & - & - & - \\
\hline 3 & IMP & Present & Multilayer & ++ & ++ & + & Present & Single cells & - & - & - \\
\hline 4 & IMP & Present & Multilayer & - & +++ & - & Present & Single cells & + & - & - \\
\hline 5 & IMP & - & Monolayer & +++ & + & - & Present & Monolayer & ++ & + & - \\
\hline 7 & IMP & - & Multilayer & +++ & ++ & + & Present & Single cells & ++ & - & + \\
\hline 8 & IMP & - & Multilayer & ++ & ++ & - & Present & None & - & - & - \\
\hline 9 & IMP & - & Multilayer & ++ & ++ & - & Present & None & - & - & - \\
\hline 10 & IMP & Present & Multilayer & +++ & ++ & - & Present & Single cells & + & - & - \\
\hline 11 & IMP & - & Multilayer & ++ & ++ & - & Present & None & - & - & - \\
\hline 12 & VMTS & - & Multilayer & +++ & ++ & - & Present & None & - & - & - \\
\hline
\end{tabular}

*Cell proliferation at the vitreal side of the inner limiting membrane if present.

FLSC, fibrous long spacing collagen; ILM, inner limiting membrane; IMH, idiopathic macular hole; IMP, idiopathic macular pucker; NFC, newly formed collagen; NVC, native vitreous collagen; VMTS, vitreomacular traction syndrome.

removal was conducted using an end-gripping forceps. Surgery was completed by gas tamponade in $\mathrm{IMH}$ patients $\left(15 \% \mathrm{C}_{2} \mathrm{~F}_{6}\right.$ gas mixture) after fluid-air exchange, and patients were encouraged to keep a face-down positioning for 4 days.

The ERM and ILM specimens harvested during vitrectomy were immediately placed into phosphate-buffered 4\% glutaraldehyde solution for fixation. Specimens were processed for light and electron microscopy as reported previously. ${ }^{10}$ Analysis and imaging of about 50 ultrathin sections per specimen were performed using a Zeiss light microscope and a Zeiss EM 9 S-2 electron microscope (Zeiss, Jena, Germany). The morphological analysis was blinded and focused on both the vitreal and the retinal side of the ILM if present which included the documentation of distribution and type of cells and collagen. The ultrastructural features of cells and extracellular matrix components were characterised as previously listed, and retinal debris at the ILM was rated using a four-point grading scale as previously reported. ${ }^{11} 12$

\section{RESULTS}

Twenty-six surgical specimens from 18 women and eight men were included in this study, corresponding to 16 right eyes and 10 left eyes. The average age at time of surgery was 70 years (range 62-80 years). In all eyes, two specimens per eye were analysed. Table 1 demonstrates the ultrastructural findings of all patients.

The first surgical specimen of all eyes, which showed no staining with BBG (figure 1), revealed masses of cells and collagen (figure 2A). In three specimens from eyes with IMP, we found fragments of the ILM, which were removed along with the first, unstained, surgical specimen (figure 2A). Cell proliferations were distributed as cell multilayers on irregularily folded collagen strands (figure $3 \mathrm{~A}$ ). Myofibroblasts were the predominating cell type. They were characterised by a lobulated cell nucleus and aggregates of 5-7 $\mathrm{nm}$ subplasmalemmal cytoplasmatic filaments with fusiform densities. Cell proliferations often showed polarisation with prominent microvillous processes and interposition of native vitreous collagen as well as newly formed collagen. Fibrils of native vitreous collagen were regularly arranged with a diameter of 10-16 nm, whereas fibrils of newly formed collagen were irregularly distributed with a diameter of more than $16 \mathrm{~nm}$. In three specimens, fibrous long spacing collagen was seen that presented with a symmetrical cross-striation of periodicity. In about half of all eyes with IMP, collagen fibrils were densely packed similar to a basal lamina-like strand (figure 3B).

The second surgical specimen was removed sequentially after a second administration of BBG and demonstrated good staining characteristics intraoperatively. The histological evaluation of these specimens confirmed that all these membranes corresponded to the ILM (figure $2 \mathrm{~B}$ ). In five cases, some remaining collagen fibrils were found at the vitreal side of the ILM, mainly described as native vitreous collagen (figure 3C). When collagen was found at the ILM, cell proliferation was always present. In seven eyes, the ILM specimens were seen with minor cell proliferations such as single cells or cell monolayers (figure 3D). Myofibroblasts, fibroblasts and fibrous astrocytes were present. Fibroblasts were characterised by abundant rough endoplasmatic reticulum, prominent golgi complexes and the absence of intracytoplasmatic filaments. Fibrous astrocytes were characterised by masses of intracytoplasmatic intermediate-type filaments and polarisation with basement membrane production. Of note, cell or collagen remnants at the vitreal side of the ILM did not interfere with the positive staining during surgery. In six of all eyes, ILM specimens were devoid of cells and collagen.

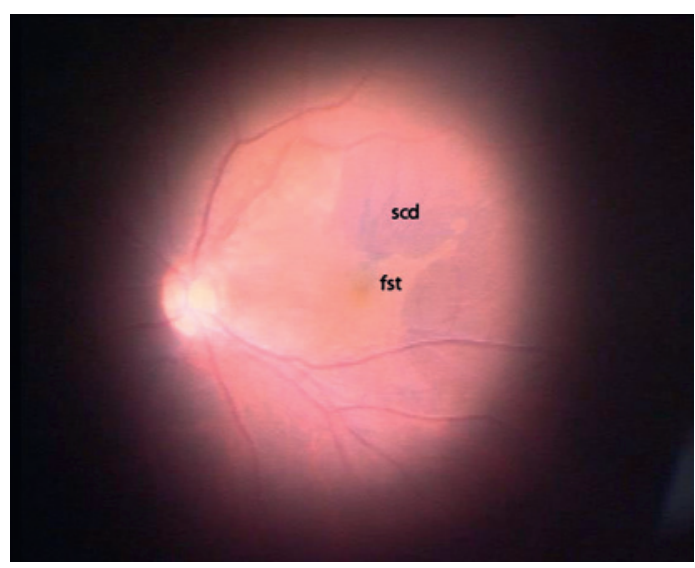

Figure 1 Intraoperative observation of unstained tissue (fst) removed first, and stained tissue (scd) sequentially removed presenting with a blue-coloured appearance. 
Figure 2 Light micrographs of unstained $(A)$ and stained $(B)$ tissue removed consecutively by brilliant blue G-assisted peeling in macular surgery for macular pucker. (A) Cell proliferations (arrows) irregularly distributed as cell multilayers on folded collagen strands (arrowheads). (B) Internal limiting membrane (asterisk) seen devoid of cells and collagen with a characteristic undulated retinal side (arrow) and a smooth vitreal side (arrowhead). Original magnification: $\times 1000$.
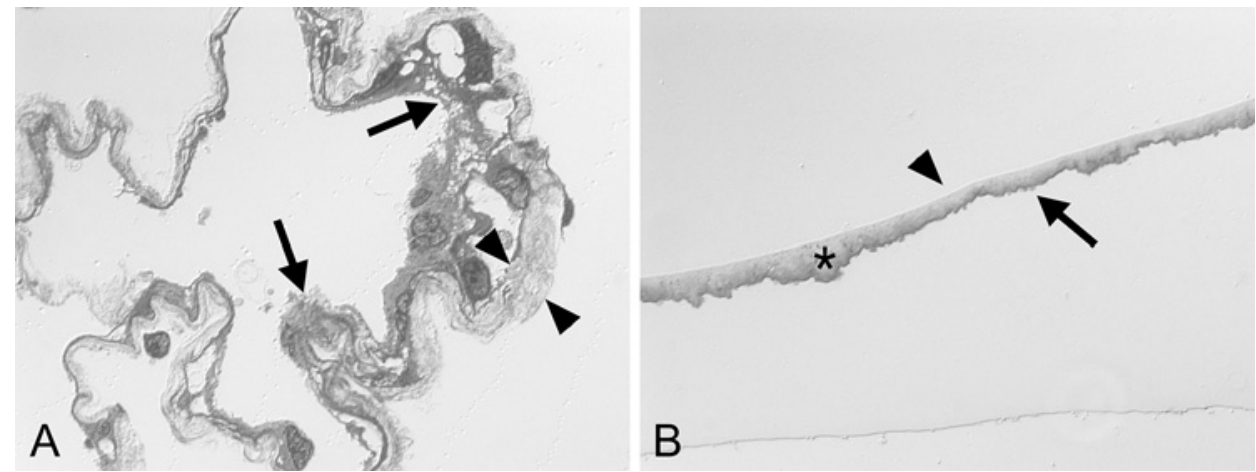

Retinal debris was solitarily distributed as small round cell fragments directly adjacent to the retinal side of the ILM.

\section{CONCLUSION}

Our findings indicate that epiretinal membrane formation reduces sufficient visualisation of the ILM during BBG-assisted macular surgery, and that BBG does provide a selective staining of the ILM. In this study, ERM specimens showed intraoperatively no staining with BBG, despite the presence of ILM fragments in some of the ERM specimens, because massive epiretinal cell and collagen proliferation prevented a positive staining with BBG independent from the diagnosis. After having peeled the unstained ERM following the initial administration of BBG, residual collagen fibrils and some epiretinal cells were seen to remain adherent at the ILM. However, ILM remnants could be visualised reliably using BBG, even if a small amount of cells and collagen fibrils adhered to the vitreal side of the ILM.

From previous investigations we have learnt that the ILM is often only partly removed along with an ERM. ${ }^{13}$ Remaining ILM fragments have been associated with recurrent ERM formation. ${ }^{14-16}$ Therefore, a thorough removal of the ILM is crucial for anatomical and functional long-term success in order to remove the scaffold for further cell proliferation and contraction.

We believe that our findings have implications for the surgical technique of tractional maculopathies. In this context, first, we question whether it is necessary to apply vital dyes to stain and remove epiretinal tissue. Second, in epiretinal membrane surgery, when the surgeon intents to additionally remove the ILM, it has to be questioned whether double staining procedures are indeed
Figure 3 Transmission electron micrographs of unstained $(A, B)$ and stained $(C, D)$ tissue removed consecutively by brilliant blue G-assisted peeling in macular surgery for macular pucker. (A) Myofibroblasts characterised by a lobulated cell nucleus and aggregates of $5-7 \mathrm{~nm}$ subplasmalemmal cytoplasmatic filaments dominated in cell proliferations on masses of newly formed collagen (arrow). Newly formed collagen was irregularly packed with a fibril diameter of more than $16 \mathrm{~nm}$. (B) Collagen fibrils, often found densely packed similar to a basal lamina like strand (arrow). (C) Single collagen fibrils (arrow),

distributed at the vitreal side of the ILM (asterisk). (D) Native vitreous collagen fibrils (arrow), with a diameter of $10-16 \mathrm{~nm}$, interposed between single epiretinal cells and the ILM (asterisk). Retinal debris was mostly seen as small round cell fragments (arrowhead) at the undulated side of the ILM. Original magnification: $(A) \times 1800$; $(B-D)$ $\times 4800$.
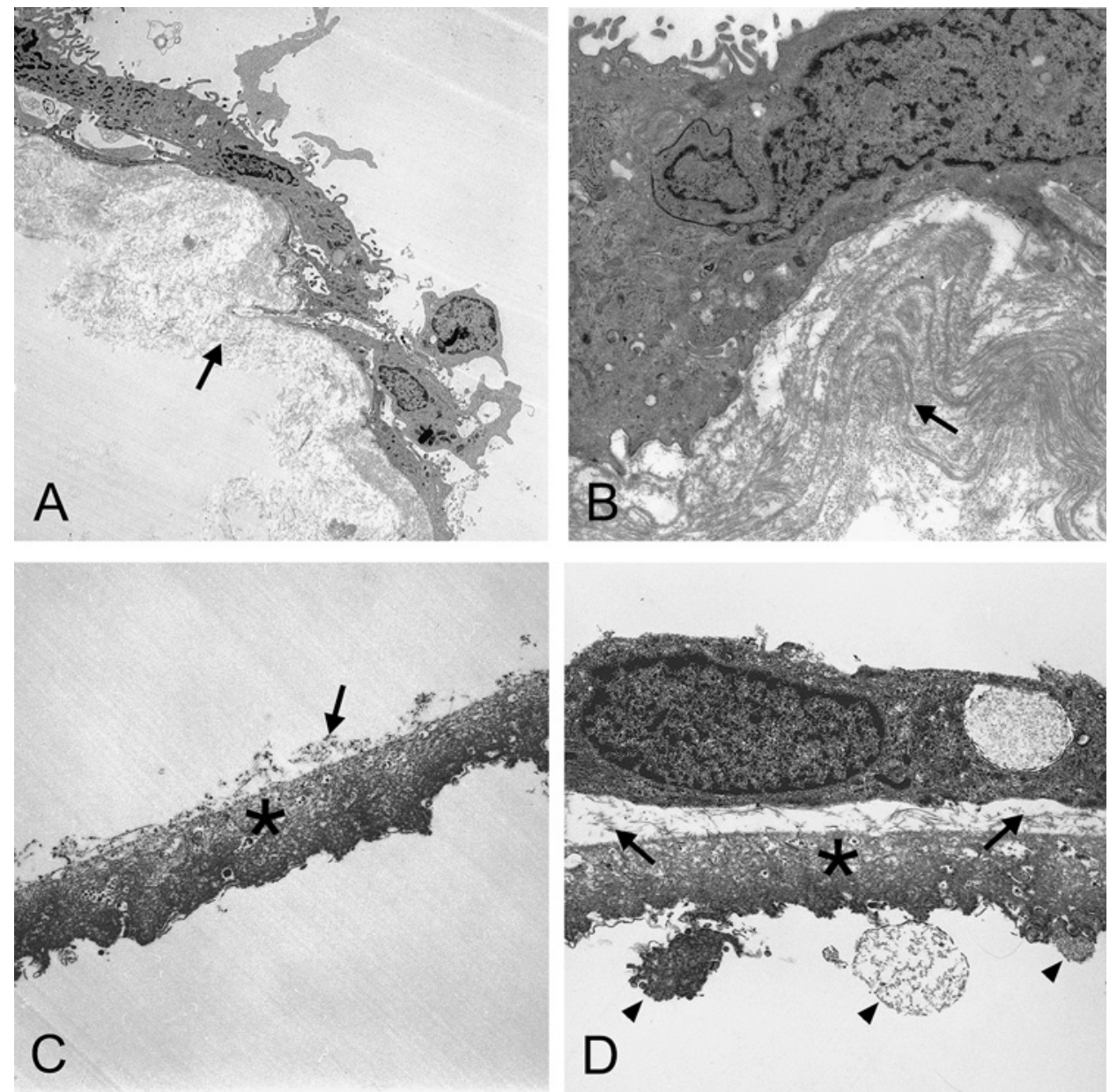
mandatory as described previously. ${ }^{17}$ We conclude from the present clinicopathological correlation with BBG that it may be sufficient to remove epiretinal tissue without dye assistance. The use of the dye could be limited to visualise ILM remnants, to peel them off safely, thereby removing cells and collagen adherent to the vitreal side of the ILM that would otherwise have been left behind at the macula. In other words, BBG helps to identify the ILM and facilitates its complete removal.

In general, the application of vital dyes should be limited to situations when they are very helpful and necessary. The results provided in this study represent a step in that direction.

\section{Competing interests None.}

Patient consent Obtained.

Ethics approval Ethics approval was provided by the local institutional review board and ethics committee of the Ludwig-Maximilians-University Munich.

Provenance and peer review Not commissioned; externally peer reviewed.

\section{REFERENCES}

1. Enaida H, Hisatomi T, Goto $Y$, et al. Preclinical investigation of internal limiting membrane peeling and staining using intravitreal brilliant blue G. Retina 2006:26:623-30.

2. Enaida $\mathbf{H}$, Ishibashi T. Brilliant blue in vitreoretinal surgery. Dev Ophthalmol 2008;42:115-25.

3. Haritoglou C, Thaler S, Kampik A, et al. Vital dyes in vitreoretinal surgery. Current application concepts. Ophthalmologe 2009;106:7-10.

4. Henrich PB, Haritoglou C, Meyer P, et al. Anatomical and functional outcome in brilliant blue $\mathrm{G}$ assisted chromovitrectomy. Acta Ophthalmol. Published Online First: 23 April 2009. doi:10.1111/j.1755-3768.2008.01477.
5. Kawahara S, Hata Y, Miura $\mathrm{M}$, et al. Intracellular events in retinal glial cells exposed to ICG and BBG. Invest Ophthalmol Vis Sci 2007;48:4426-32.

6. Lüke M, Januschowski K, Beutel J, et al. Electrophysiological effects of brilliant blue $\mathrm{G}$ in the model of the isolated perfused vertebrate retina. Graefes Arch Clin Exp Ophthalmol 2008;246:817-22.

7. Remy M, Thaler S, Schumann RG, et al. An in vivo evaluation of brilliant blue $\mathrm{G}$ in animals and humans. Br J Ophthalmol 2008;92:1142-7.

8. Schumann RG, Remy M, Grueterich M, et al. How it appears: electron microscopic evaluation of internal limiting membrane specimens obtained during brilliant blue $\mathrm{G}$ assisted macular hole surgery. Br J Ophthalmol 2008;92:330-1.

9. Kifuku K, Hata $Y$, Kohno R-I, et al. Residual internal limiting membrane in epiretinal membrane surgery. Br J Ophthalmol 2009:93:1016-19.

10. Schumann RG, Schaumberger MM, Rohleder $M$, et al. Ultrastructure of the vitreomacular interface in full-thickness idiopathic macular holes: a consecutive analysis of 100 cases. Am J Ophthalmol 2006;141:1112-19.

11. Gandorfer A, Rohleder R, Grosselfinger $\mathrm{S}$, et al. Epiretinal pathology of diffuse diabetic macular edema associated with vitreomacular traction. Am J Ophthalmol 2005; 139:638-52.

12. Schumann RG, Gandorfer A, Priglinger SG, et al. Vital dyes for macular surgery: A comparative electron microscopy study of the internal limiting membrane. Retina 2009;29:669-76

13. Kampik A, Green WR, Michels RG, et al. Ultrastructural features of progressive idiopathic epiretinal membrane removed by vitreous surgery. Am J Ophthalmol 1980;90:797-809.

14. Kwok AK, Lai TY, Yuen KS. Epiretinal membrane surgery with or without internal limiting membrane peeling. Clin Exp Ophthalmol 2005;33:379-85.

15. Maguire AM, Smiddy WE, Nanda SK, et al. Clinicopathologic correlation of recurrent epiretinal membranes after previous surgical removal. Retina 1990;10:213-22.

16. Shimada H, Nakashizuka $H$, Hattori $T$, et al. Double staining with brilliant blue $\mathrm{G}$ and double peeling for epiretinal membranes. Ophthalmology 2009;116:1370-6.

17. Stalmans $\mathbf{P}$, Freon EJ, Parys-Van Ginderdeuren $\mathrm{R}$, et al. Double vital staining using Trypan Blue and infracyanine green in macular pucker surgery. $\mathrm{Br} \mathrm{J}$ Ophthalmol 2003;87:713-16 


\section{Sequential epiretinal membrane removal with internal limiting membrane peeling in brilliant blue G-assisted macular surgery}

Ricarda G Schumann, Arnd Gandorfer, Kirsten H Eibl, et al.

Br J Ophthalmol 2010 94: 1369-1372 originally published online July 31, 2010

doi: $10.1136 /$ bjo.2010.183210

Updated information and services can be found at:

http://bjo.bmj.com/content/94/10/1369.full.html

These include:

References This article cites 16 articles, 5 of which can be accessed free at: http://bjo.bmj.com/content/94/10/1369.full.html\#ref-list-1

Email alerting Receive free email alerts when new articles cite this article. Sign up in service the box at the top right corner of the online article.

\begin{tabular}{cc}
$\begin{array}{c}\text { Topic } \\
\text { Collections }\end{array}$ & $\begin{array}{c}\text { Articles on similar topics can be found in the following collections } \\
\text { Retina (1328 articles) } \\
\text { Ophthalmologic surgical procedures (1054 articles) }\end{array}$ \\
\hline
\end{tabular}

Notes

To request permissions go to:

http://group.bmj.com/group/rights-licensing/permissions

To order reprints go to:

http://journals.bmj.com/cgi/reprintform

To subscribe to BMJ go to:

http://group.bmj.com/subscribe/ 